Supporting Information for

\title{
Probing the Degradation Chemistry and Enhanced Stability of 2D Organolead Halide Perovskites
}

Bryan R. Wygant, ${ }^{a}$ Alexandre Z. Ye,${ }^{b}$ Andrei Dolocan, ${ }^{c}$ Quyen Vu, ${ }^{b}$ David M. Abbot,${ }^{b}$ C. Buddie

$$
\text { Mullins }^{a, b, c^{*}}
$$

${ }^{a}$ Department of Chemistry, ${ }^{b}$ McKetta Department of Chemical Engineering, ${ }^{c}$ Texas Materials

Institute; University of Texas at Austin, Austin, TX 78712

*corresponding author: mullins@che.utexas.edu 


\title{
Table of Contents
}

\author{
Page \\ $\underline{\text { Content }}$ \\ S3 \\ Supplemental Discussion S1: Hydrophobicity of quasi- \\ 2D perovskite materials \\ Supplemental Discussion S2: Spectroscopic and \\ S5 \\ Physical effects of nBA-MAPI Disproportionation \\ S7 \\ Supplemental Discussion S3: Water diffusion and \\ damage at the perovskite/PEDOT:PSS interface \\ S9 \\ Supplemental Figures S1-19, Table S1 \\ S29 \\ Supplemental References
}


Supplemental Discussion S1: Hydrophobicity of quasi-2D perovskite materials

Density functional theory (DFT) studies have shown that the enhanced van der Waals interactions of the longer alkyl chain on the A' linker molecule (n-butylammonium, or $\mathrm{nBA}$, here and phenyethylammonium in previous work) help to stabilize quasi-2D perovskite phases with lower $n .{ }^{1}$ This increases the desorption energy of the molecule relative to methylammonium (MA), and acts to stabilize the otherwise unprotected $\mathrm{PbI}_{2}$ surface. While not directly cited in this work, it is also reasonable that the relative hydrophobicity of the A' alkyl chain, which is related to the relative strength of the van der Waals interactions, will also decrease the interaction of water at the exposed surface. A stable, low- $n$ nBA-MAPI phase would have a proportionally higher quantity of hydrophobic nBA relative to a higher- $n$ phase, potentially making the effect more pronounced. In 2D/3D MAPI systems treated with both butylammonium and octylammonium, such an increase in hydrophobicity with the presence of long alkyl chains has previously been observed. ${ }^{2}$ The authors observed an increasing contact angle as the length of the alkyl chain on the alkylammonium cation increased (methyl to butyl to octyl), and this was positively correlated with increased stability in both XRD measurements and device performance. Other works have also mentioned the increased hydrophobicity of these molecules, but usually only note in passing that it protects the interior of the perovskite from water diffusion by inhibiting the migration of water into the film interior. ${ }^{3-5}$

Thus, while the effect of hydrophobicity is undoubtedly important, it is difficult to fully disentangle from the increased thermodynamic stability of the alkyl chain-rich low- $n$ phases. Furthermore, hydrophobicity will almost certainly be directly impacted by the crystallographic orientation of the quasi-2D perovskites in the films and their predominant surface; this can be demonstrated in two distinct cases. In Case I, hot casting of the quasi-2D perovskites films has been shown by us and others ${ }^{5}$ to create films where the perovskite sheets are stacked orthogonally to the substrate; each sheet is expected to terminate in a methylammoniumterminated edge and possess long planes of A' perpendicular to the substrate (Figure S6b). Conversely, the theoretical calculations by Quan et al. were performed on surfaces that would be terminated almost exclusively in A' linker molecules, and thus such crystals would be oriented parallel to the substrate (Case II). An example of this can be found in a work studying the thermal stability of such perovskites treated to create a $2 \mathrm{D} / 3 \mathrm{D}$ structure similar to the system that we present; the authors propose that the crystals grains will be coated in a uniform layer of the linker molecule. ${ }^{6}$

Assuming idealized surfaces for Case I and II on a pure 2D perovskite, and ignoring low- $n$ phase stability and our proposed disproportionation momentarily, diffusion of water into the films would almost certainly occur at very different rates. In Case I, water would diffuse more slowly along, or completely fail to penetrate into, the long planes of nBA molecules perpendicular to the substrate, as has been previously suggested. ${ }^{4,5}$ Unfortunately, it seems equally probable that water would then simply penetrate into the depth of the film through the relatively unprotected methylammonium-terminated sheet edges, offsetting this advantage. Case II would prevent this 
attack from the top edge almost entirely, offering a hydrophobic surface with which water would be unlikely to interact strongly. Extrapolating from Case I, moisture related damage would then need to travel laterally from the edge of the film to the center, and thus the apparent rate of degradation would be much slower when compared to Case I, as the distance from edge-tocenter is much larger than from surface-to-center. The trade-off, however, is that this surface coating of nBA would be expected slow charge transfer out of the perovskite, and indeed, may explain some of the slow carrier mobility measured in such films. ${ }^{5,7}$ Work on nanoparticle-based systems has shown that charge transfer will still occur, but would likely be slower than in Case I. ${ }^{8}$ In summary, in Case I water diffusion would be inhibited in the lateral plane of the perovskite, but unimpeded in the vertical direction; in Case II, diffusion of moisture would be limited in the vertical plane, but unhindered in the lateral plane of the film.

Finally, with these two examples in hand, we can attempt to apply these principles to our particular 2D and 2D/3D systems and predict what effect hydrophobicity may have. Starting with the 2D/3D system, based on the ToF-SIMS profile in Figure S17c and previous work on similar systems, ${ }^{6}$ there is a sub-10 nm layer of nBA-MAPI at the surface of the film, oriented as in Case II, and a thin surface coating of nBA on some portion of the 3D MAPI grain boundaries throughout the film depth. Together, this would inhibit water diffusion through the cell both laterally and vertically. For the 2D film, XRD evidence suggests that the dominant orientation for the film must be similar to that in Case I, and that while water diffusion through the film would be lower than in pure 3D MAPI, it will still suffer from attacks to the vertical sheet edges. We postulate that a thin layer of nBA could substitute for the MAPI on the extreme outer edge of the perovskite sheet, increasing stability as in Case II, but there is no way to determine this definitively. Instead, in such a case, it is very likely that the increased thermodynamic stability is imparting the increased resistance to moisture. 


\section{Supplemental Discussion S2: Spectroscopic and physical effects of nBA-MAPI}

\section{Disproportionation}

PL spectroscopy has been used to probe the existence and relative location of various $n$-phases within quasi-2D perovskite thin films, ${ }^{79-12}$ but care must be taken to account for the electronic band structure of these materials. Due to the effects of quantum confinement on the perovskite chromophore, adjacent $(n)$ and $(n-1)$ perovskites will form type II heterojunctions, leading to rapid energy transfer from low- to high- $n$ species. ${ }^{7,910}$ Steady-state PL experiments show that the front of hot-cast perovskite films, such as those used in this study, have a predominately high- $n$ character, and a back with predominately low- $n$ character; this allows electrons and holes to be separated within the film under illumination. ${ }^{10}$ Transient absorption (TA) and time-resolved PL of the films show that the typical steady-state spectra hide the presence of low- $n$ species at or near the surface, however. ${ }^{7,9}$ Sargent and coworkers have shown that the PL spectra of these films at sufficiently short time scales ( $>5 \mathrm{~ns}$ ) show the presence of $n=2,3$, and 4 species, but that due to the type II band alignment of the perovskites, photogenerated carriers produced in the low- $n$ layers are rapidly shuttled to the bulk $2 \mathrm{D}$ perovskite phase. There, they eventually undergo radiative recombination via PL and produce the characteristic steady-state spectrum with a single peak near $1.75 \mathrm{eV}$. Thus, in order to observe an increase in PL for $n=3$ and other intermediate $n$ phases that we see in our degraded nBA-MAPI film (Figure 3c), something must be inhibiting the migration of carriers in our film.

To investigate if film topography could have an effect on this inhibition, we probed the surface of the nBA-MAPI film using atomic force microscopy (AFM) before and after exposure. For the fresh film, we observe a relatively flat surface (RMS $23.9 \mathrm{~nm}$ ) composed of flat crystal grains ranging from $100 \mathrm{~nm}$ to $1 \mu \mathrm{m}$ in diameter (Figure S11a). Based upon the work of Yuan et al. it is likely that these grains may consist of a variety of different $n$-phases, or may themselves each be a separate phase, ${ }^{9}$ but together they represent a disordered layer with an inhomogeneous energy surface (Figure S11c,e). After 8 hours of exposure to humidity, the surface looks remarkably similar on the whole, but now also features distinct crystal-like structures which appear to be growing normal to the surface (Figure S11b). This orientation and their small size may explain why they are not clearly indicated by the XRD in Figure S5. While many are visible at the site of grain boundaries, there are a number of crystals that appear to have grown from within the center of the larger perovskite grains. The crystals have an average thickness of $110 \mathrm{~nm}$, range in length from $100 \mathrm{~nm}$ to almost a micron, and generally project slightly from the surface of the film (>20 nm). Regarding the identity of these new structures, bulk quasi-2D perovskite single crystals are known to crystalize as large flat plates, ${ }^{13}$ but more importantly, the morphology of these new structures is very similar to that observed by Li et al. when they grew low- $n$ perovskite crystals on the surface of a $\mathrm{PbI}_{2}$ film. ${ }^{12}$ In agreement with this, the WAXS spectra of an nBA-MAPI film (Figure S12b,c) exposed to moisture for 8 hours shows the formation of clear rings near $q_{\mathrm{y}}=0.6 \AA$, which we can index to the $n=4(060)$ and $n=2(020)$ reflections. ${ }^{13}$ The appearance of these rings in the WAXS coincides with the formation of the 
randomly oriented platelet-structures in the AFM, making it very likely that these structures are composed of low- $n$ nBA-MAPI phases. Based on these results, we hypothesize that as the nBAMAPI degrades, the surface undergoes some degree of structural reorganization to reduce entropy in the film and produces discreet structures composed of low- $n$ nBA-MAPI perovskite phases. Concurrently, some areas of the initial disordered layer are preserved, possibly growing in thickness as degradation slowly proceeds toward the interior of the film (Figure S11d). We propose this degraded surface layer likely acts as a protective coating which slows this degradation of the bulk perovskite film, however, in agreement with our electrochemical results. Together, these structural and crystallographic changes are consistent with the enhanced stability of low- $n$ perovskite phases first reported by Sargent and coworkers. ${ }^{1}$

These discreet low- $n$ structures may also help explain the increased PL intensity for low- $n$ species that we observe in our steady state experiments. As these structures form and likely degrade from the outside in, they would naturally create structures with a low- $n$ surface surrounding a higher- $n$ interior. Based on reported band positions and relative band structures for these quasi-2D perovskites, this could give rise to one of two distinct quantum well-like band structures within these platelets. ${ }^{14,15}$ In the first case, given the band structure first reported by Cao et al. for $n$-butylammonium-based quasi-2D perovskites, ${ }^{14}$ the various adjacent $n$-phases would form a series of type II heterojunctions. As can be seen in Figure S11f, carriers photogenerated inside the structure would tend to flow downhill to the high- $n$ phases at the core (here composed largely of $n=3$ and 4) and become trapped, eventually recombining and emitting PL at the wavelengths we observe in Figure 3b. Additionally, 3D MAPI produced as a byproduct of degradation at the outside of these structures could likewise collect electrons from neighboring phases, producing the increase in 3D MAPI PL that we observe. Meanwhile, holes would migrate to the low- $n$ edges of the structures and become caught in shallow trap states. ${ }^{7}$ Once trapped, they would eventually recombine by largely non-radiative trap-assisted processes, as previously observed for most trapped carriers in organolead halide perovskites. ${ }^{16}$ Transfer of electrons to high- $n$ phases and holes to low- $n$ phases has been observed in several studies using ultrafast transient absorption spectroscopy, ${ }^{7,9,12,17-20}$ and such transfer agrees well with the relative band alignments these groups propose using ultraviolet photoelectron spectroscopy (UPS).

In the second case, recent work from Kahn et al. has suggested that when an $n$ and $n+1$ phase are adjacent to each other, a type I heterojunction forms instead of the previously proposed type II heterojunction. ${ }^{15}$ From density functional theory (DFT), they find that these perovskites possess a low density of states near the valence band maximum that makes accurate determination of the exact position of the valence band maximum and conduction band minimum difficult using UPS alone. ${ }^{15,21}$ Further, they show that the two band edges are composed primarily of $\mathrm{Pb}$ and I states, indicating that neither the butylammonium spacers nor the methylammonium inside the perovskite lattice will have a significant impact on the electronic structure near the band edges. ${ }^{15,21,22}$ Together, this suggests altogether different band structure will form within the 
platelets at the surface, presented here in Figure S11g. In this configuration, electrons will still collect in the interior of the crystals, as in the previous case, but holes will no longer collect at the outer edges of the platelet. Instead, the holes will also transfer to the higher- $n$ phases within of the interior along with the electrons. This would provide a ready source of both electrons and holes for radiative recombination, and agrees with the increased PL we observe for our films. While more investigation is needed to definitively determine whether a type I or type II heterojunction is formed in these quasi-2D materials, it seems probable that either band alignment would result in the electronic isolation of $n=2,3$, and 4 phases produced by degradation within the surface crystallites. This isolation prevents the extraction of photogenerated-charges into the high- $n$ phases of the bulk and allows us to observe the formation of these phases via degradation in the PL spectra of our films.

$\underline{\text { Supplemental Discussion S3: Water diffusion and damage at the perovskite/PEDOT:PSS }}$ interface

There are two interfaces at which the perovskite absorber layer can interact with the outside environment: the top where it contacts $\mathrm{C}_{60}$, and the bottom where it contacts PEDOT:PSS. Either interface could be damaged by water which has diffused there, and must be considered when assessing stability. Regarding the top $\left(\mathrm{C}_{60} /\right.$ perovskite) interface, the largest chemical changes visible in the ToF-SIMS profiles occur at this interface. As seen in Figure 6a,c, MAPI shows clear formation of new inorganic $\left(\mathrm{PbI}_{2}^{-}\right)$and organic $\left(\mathrm{C}_{2} \mathrm{~N}^{-}\right)$and $\left.\mathrm{CH}_{2}^{-}\right)$peaks here, and both the nBA-MAPI and 2D/3D MAPI show more notable peak broadening at this interface when compared to the back interface (perovskite/PEDOT). Further, when comparing the relative stabilities of the MAPI and 2D/3D MAPI devices, it is apparent that the surface treatment appears to have significantly improved the stability of this 3D perovskite. While the depth profile in Figure S17a does show some degree of penetration into the film by the $\mathrm{nBA}^{+}$along grain boundaries, the primary peak is still at the surface of the film, and thus the protection likely comes strongly from there. Finally, in the PL spectra of the nBA-MAPI film (Figure 3b), the most significant changes occur in the front-side spectra rather than the back. Thus, the experimental results strongly suggest that the top interface is of significant importance when studying degradation in these devices, and it is for this reason that we chose to focus much of our analysis on this interface.

Regarding the bottom interface with the PEDOT:PSS layer, it is both possible and probable that some degree of damage does occur here, but evidence for the extent of this damage is not as clear as for the top. ToF-SIMS depth profiles in Figure 6 show slight variations in some of the organic species at the back interface, but the changes are not as significant as those at the top. Further, as mentioned above, all changes to the inorganic species appear to occur at the upper surface, with little to no change occurring near the PEDOT. This observation may be due, in part, the relative amount of water which is traveling to each interface during exposure, which is 
related to both the type and quantity of material through which the water must diffuse. While diffusion through the $\mathrm{C}_{60}$ is expected to be slow due to the hydrophobicity of the molecule, the diffusion path itself is extremely short $(\sim 50-200 \mathrm{~nm})$. Conversely, water has been calculated to have a relatively high diffusion constant of $5 \times 10^{-6} \mathrm{~cm}^{2} / \mathrm{s}$ in PEDOT:PSS ${ }^{23}$ but must diffuse across approximately $0.75-1 \mathrm{~cm}$ to reach the active device area from the unprotected outer edge of the substrate. Thus, new water arriving at the interface would take on the order of 20 hours to travel the $1 \mathrm{~cm}$ distance from the substrate edge to the device area in the center. This is significantly slower than the rate of degradation exhibited by the 3D MAPI film, and is thus unlikely to be the source of degradation in this film, or by extension the other films.

Finally, we also note that, as previously discussed, the 3D MAPI and 2D/3D MAPI films differ only in the nBA surface treatment and yet exhibit very different electrochemical degradation rates. As the two films are thus expected to have very similar perovskite/PEDOT:PSS interfaces, this clearly suggests that this interface cannot be the dominant source of water-based degradation, and supports analysis of the top interface with $\mathrm{C}_{60}$. Further studies on alternative hole transport materials, such as $\mathrm{P} 3 \mathrm{HT}$ and $\mathrm{NiO}$ would be interesting, and may provide a more nuanced view of the choice of substrate on the moisture stability of both 2D and 3D perovskites. 


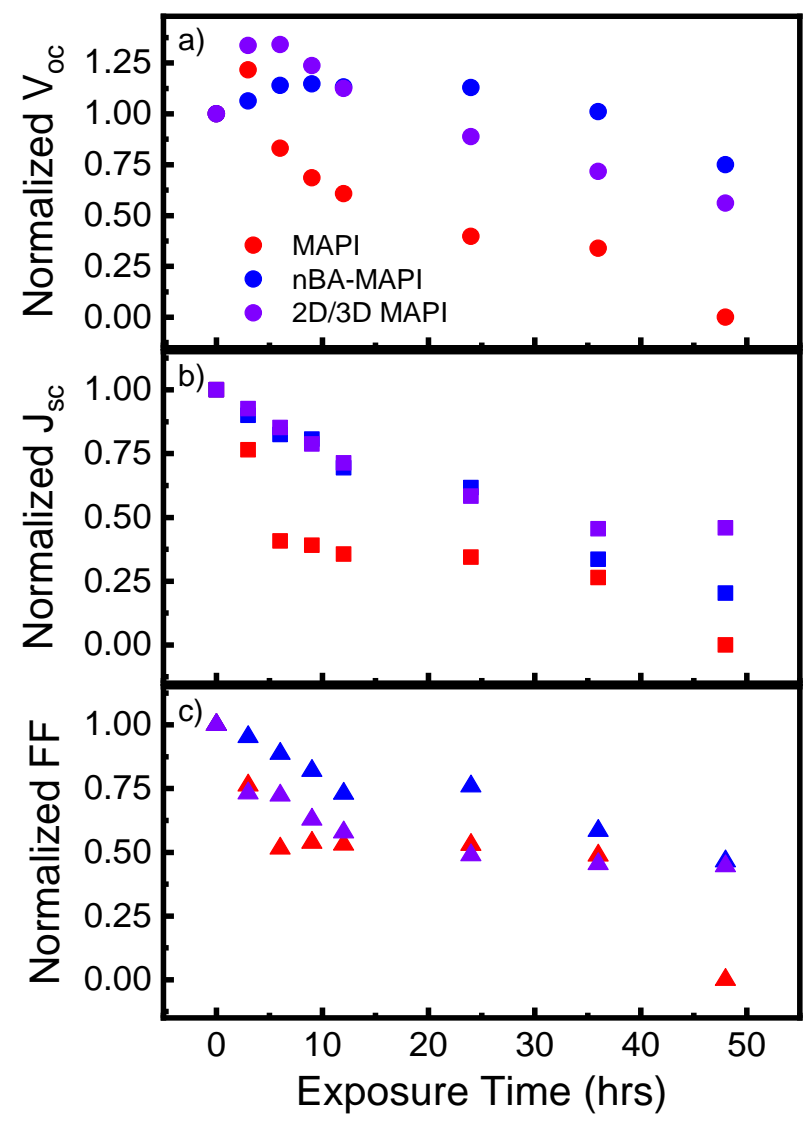

Figure S1: Normalized (a) open-circuit potential, (b) short-circuit current, and (c) fill factor values for MAPI, nBA-MAPI, and 2D/3D MAPI devices exposed to $78 \%$ relative humidity over time. For all three metrics, the 2D-containing perovskites show slower degradation. 


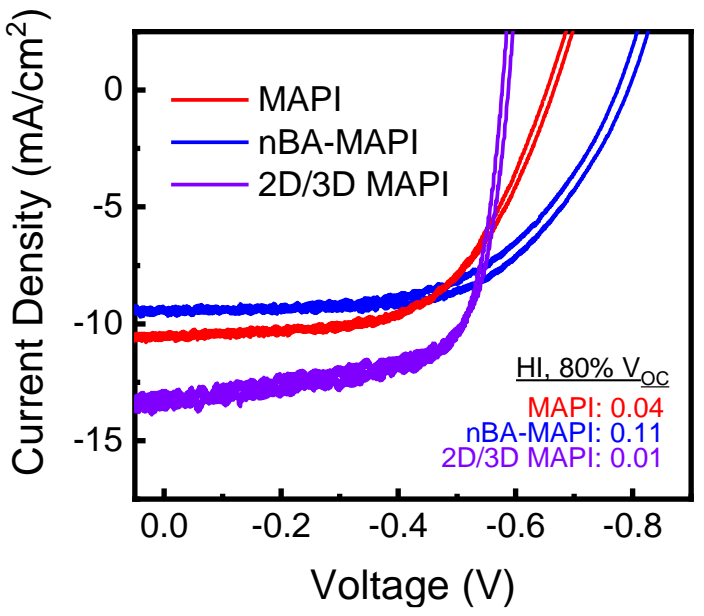

Figure S2: Cyclic voltammograms of characteristic perovskite devices before exposure to humidity showing the performance of the three types of perovskite material. A hysteresis index $(\mathrm{HI})$ at $80 \%$ of the maximum $\mathrm{V}_{\text {oc }}$ for each cell is provided, demonstrating the low degree of hysteresis between the forward and reverse scans. 

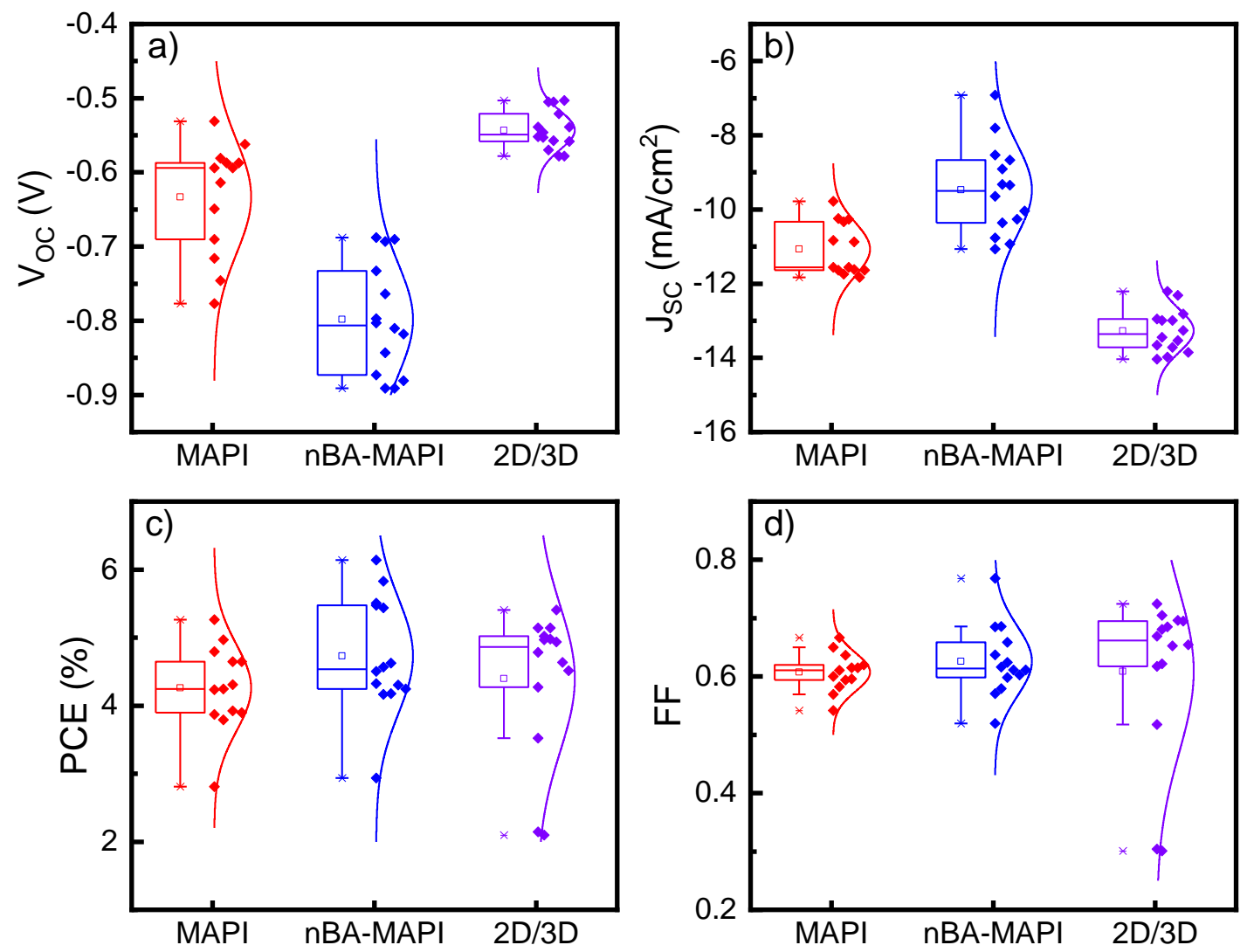

Figure S3: Statistical distributions for the a) $\mathrm{V}_{\mathrm{oc}}$, b) $\mathrm{J}_{\mathrm{sc}}, \mathrm{c}$ ) PCE, and d) FF at $\mathrm{t}=0 \mathrm{hrs}$ for all cells tested. Two partially shorted devices are responsible for the outlier values in the FF and PCE of the $2 \mathrm{D} / 3 \mathrm{D}$ devices, which would otherwise distribution similar to those for MAPI and nBA-MAPI. 


\begin{tabular}{ccccc} 
& Voc $(\mathbf{V})$ & $J_{\text {sc }}\left(\mathrm{m} \mathbf{N} / \mathrm{cm}^{2}\right)$ & PCE (\%) & FF \\
\hline MAPI & $-0.63+/-0.08(-0.746)$ & $-11.1+/-0.7(-11.6)$ & $4.26+/-0.63(5.27)$ & $0.61+/-0.03(0.61)$ \\
nBA-MAPI & $-0.80+/-0.07(-0.881)$ & $-9.47+/-1.2(-10.0)$ & $4.73+/-0.85(5.83)$ & $0.63+/-0.06(0.66)$ \\
2D/3D MAPI & $-0.54+/-0.03(-0.578)$ & $-13.3+/-0.6$ & $4.4+/-1.1(5.4)$ & $0.61+/-0.14(0.71)$ \\
\hline
\end{tabular}

Table S1: Performance metrics from electrochemical tests of MAPI, nBA$\mathrm{MAPI}$, and 2D/3D MAPI devices. Average values from a single batch of devices are reported with a standard deviation, while champion device values are included in parentheses. 


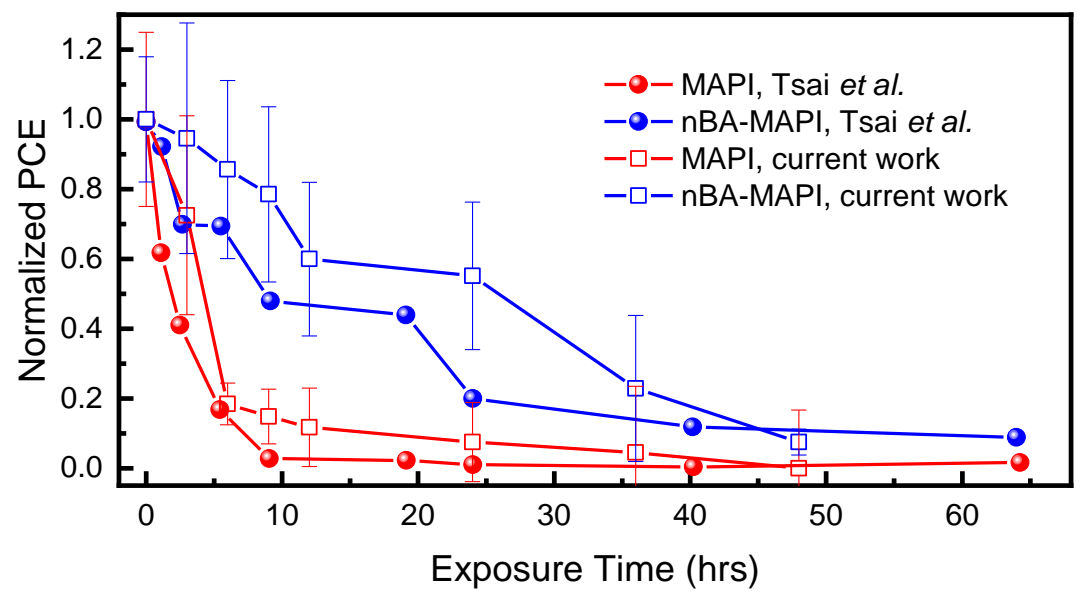

Figure S4: Normalized PCE values for MAPI and nBAMAPI devices exposed to a humid environment as reported by Tsai et al. (ref. 5), compared to similar devices reported in the current work. Within error, the devices presented here show similar degradation kinetics and trends to those reported previously, indicating the degradation process is similar in both. 


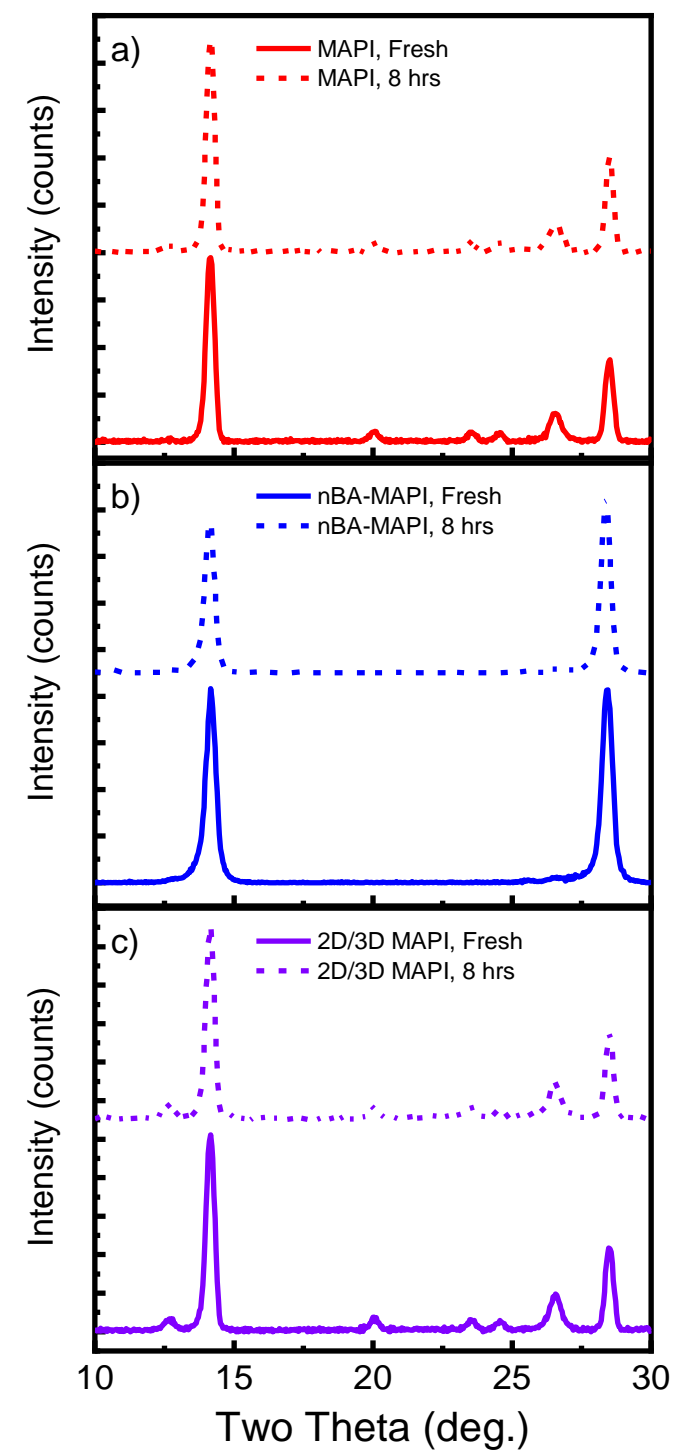

Figure S5: XRD spectra for (a) MAPI, (b) nBA-MAPI, and (c) 2D/3D MAPI films before and after exposure to $78 \% \mathrm{RH}$ for 8 hours. Little crystallographic change is observed for any of the films tested. 
a)

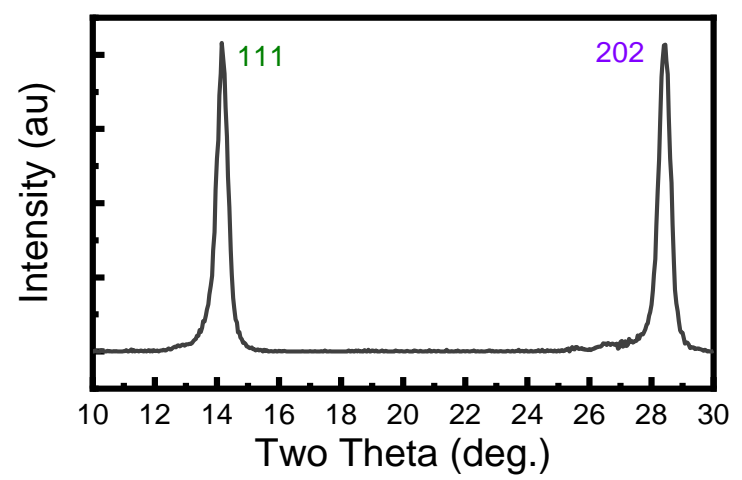

c)

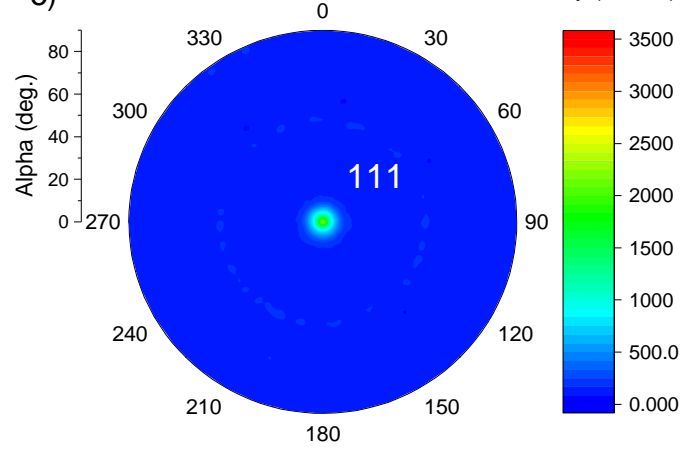

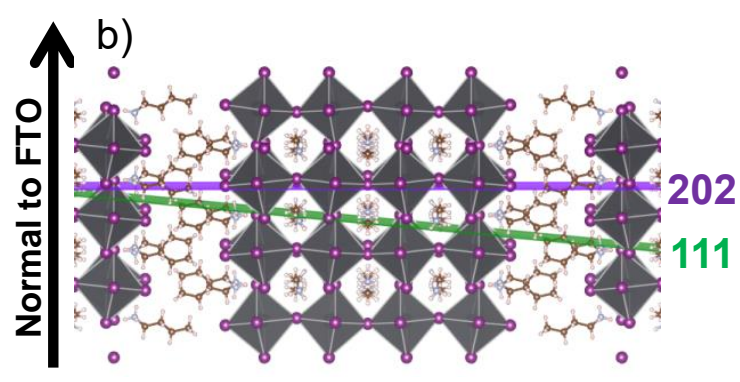

d)

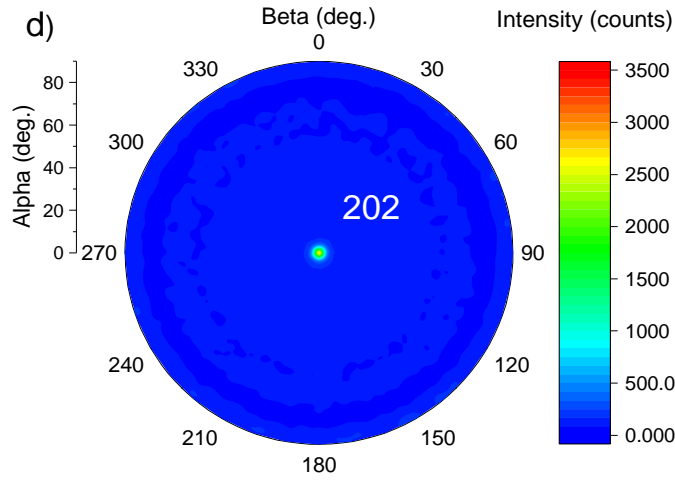

Figure S6: a) XRD spectra for a fresh nBA-MAPI film, with the 111 and 202 peaks labeled. b) graphic of an nBA-MAPI film with an orientation normal to the FTO substrate, as would be expected from a hot-cast film. Both the 111 and 202 planes have a significant horizontal component. Pole figure measurements for the 111 (c) and 202 (d) peaks, with a sharp central peak demonstrating the good agreement with the orientation of image $b$ ). 


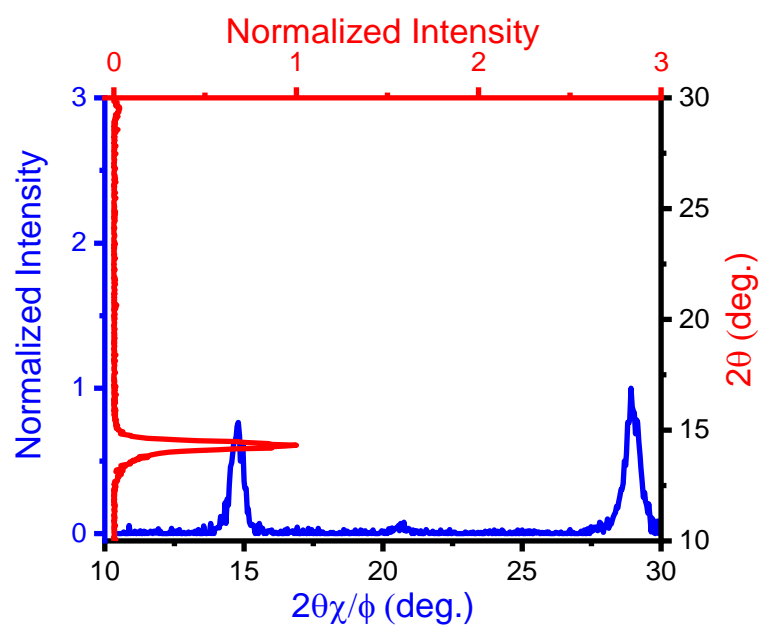

Figure S7: In-plane (y-axis) and grazing incidence (x-axis) X-ray diffraction spectra from a fresh nBA-MAPI film. The lack of the 202 peak on the $y$-axis indicates a well-ordered film. 
a)

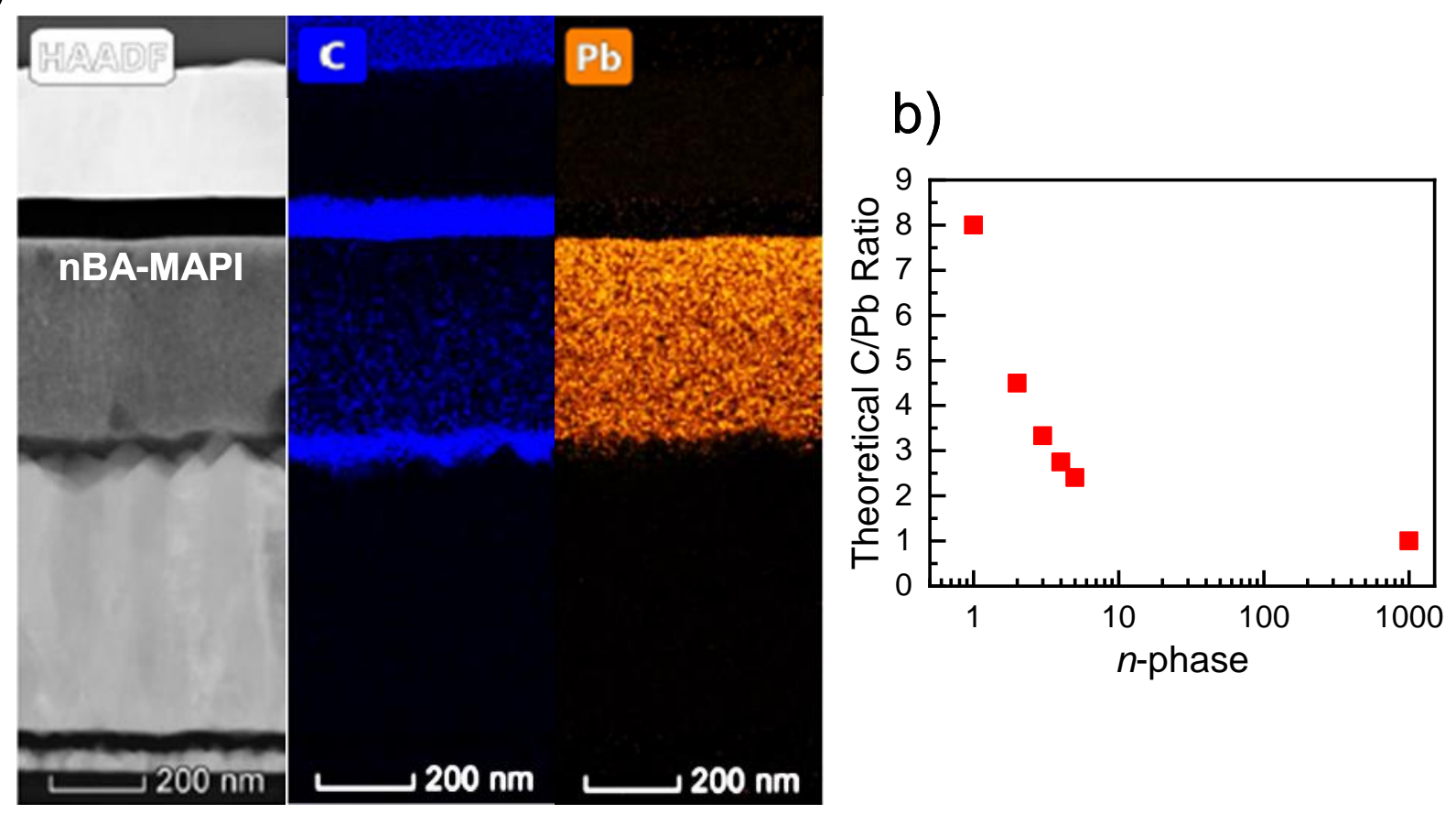

Figure S8: a) Cross-sectional HAADF (left) image of a nBA-MAPI device, with the perovskite in the center of the cross section. Accompanying EDX elemental maps of $\mathrm{C}$ (blue) and $\mathrm{Pb}$ (orange), showing a gradient in both elements as a function of depth. b) Theoretical $\mathrm{C} / \mathrm{Pb}$ elemental ratio based on the stoichiometric ratios of both elements in various $n$-phases of the nBA-MAPI perovskite, showing that the ratio would increase as $n$ decreases. 


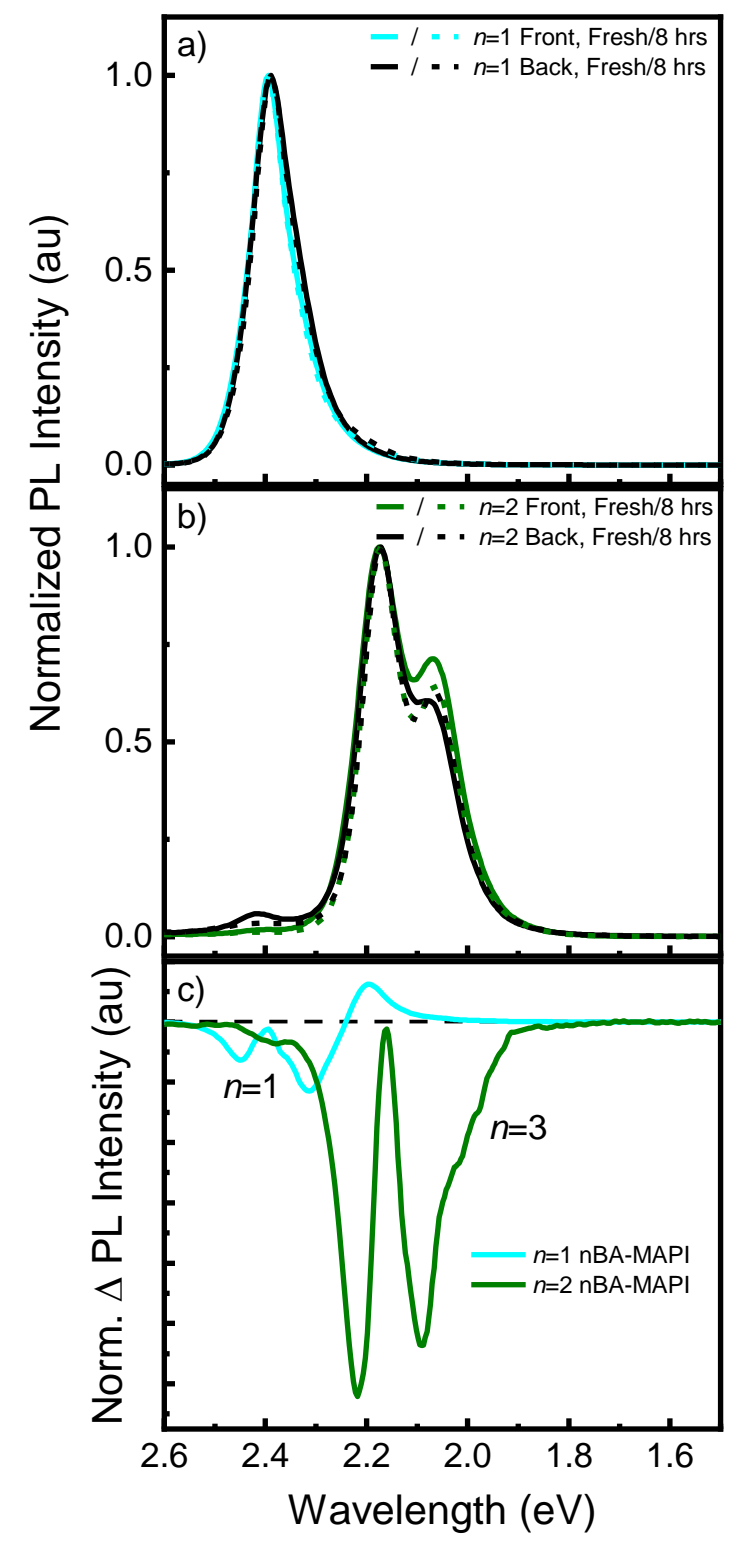

Figure S9: Normalized fluorescence spectra for the front and back of nominally a) $n=1$ and b) $n=2$ films, before and after exposure to $78 \%$ relative humidity for 8 hours. c) Normalized difference plot for the front-side spectra for both films. After exposure, the $n=1$ film shows a slight shift toward lower emission energies, while the $n=2$ film shows both the loss of $n=1$ and 3 from the surface, as well as a general peak narrowing for the $n=2$ peak. 

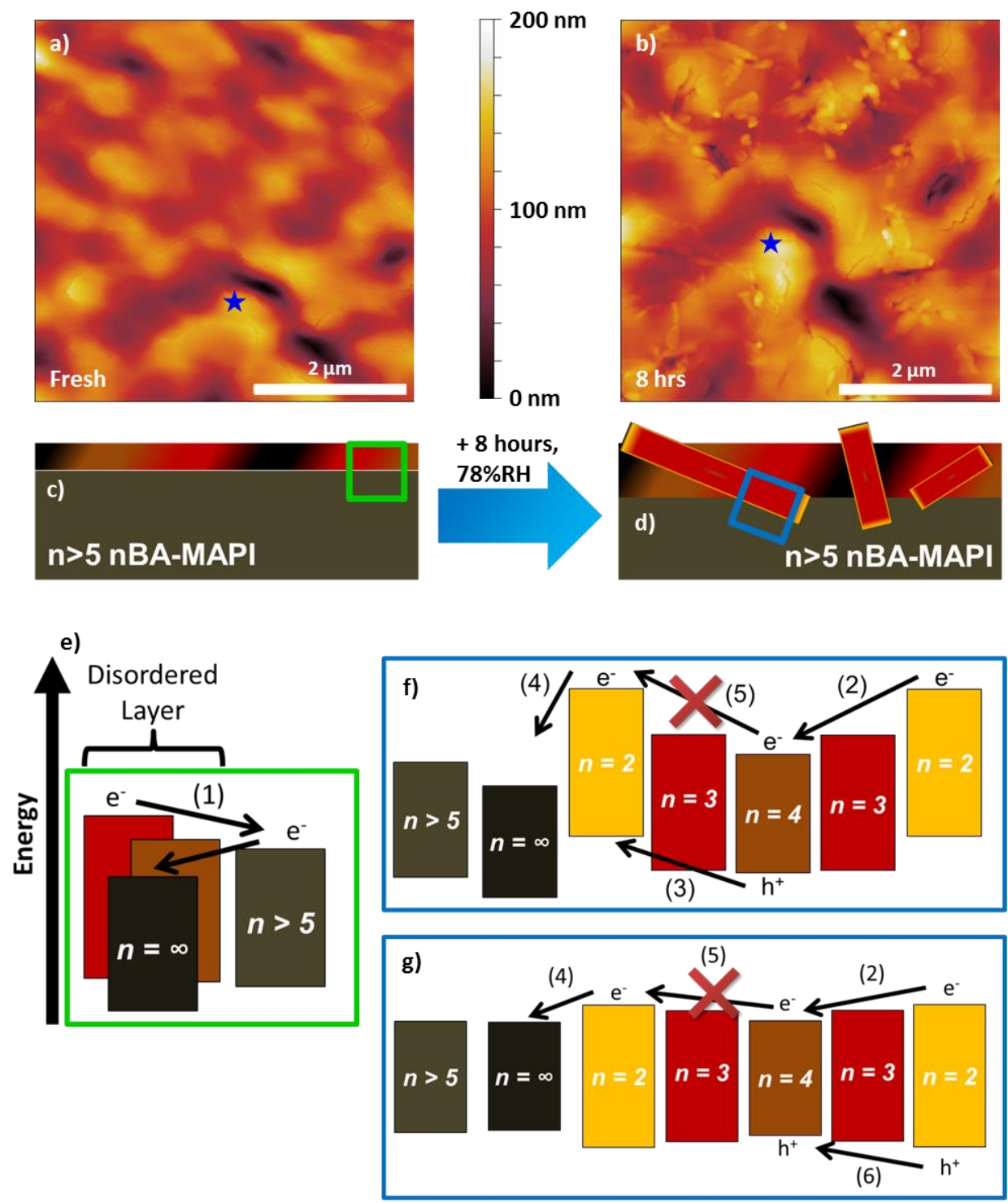

Figure S10: AFM images of an nBA-MAPI film a) before and b) after 8 hours of exposure to a $78 \% \mathrm{RH}$ environment. For reference, the blue star indicates the same location in each image. New elongated structures are observed to form on the surface of the film, and are likely discreet crystals of low- $n$ perovskite. c) Schematic of the cross section of the fresh nBA-MAPI film, showing a disordered surface layer composed of various low- $n$ phases atop a bulk of high- $n$ nBA-MAPI. d) Schematic of the cross section of the same film following moisture exposure, showing an increase in the thickness of the disordered surface, as well as the formation of the localized structures observed in b). e) Simplified energy diagram for $c$ ), showing electron transfer both from the low- $n$ phases of the surface to the bulk, and from the bulk to 3D MAPI within the disordered surface (1). f) Simplified energy diagram showing the preferential motion of electrons (2) and holes (3) generated within the type II heterojunction well structure, as well as possible charge transfer from the surface to the bulk (4). Generally, however, photogenerated electrons will be trapped in the interior, unable to escape (5). g) Simplified energy diagram for a type I heterojunction well, with similar electron transfer to the type II well. Here, hole transfer also flows to the higher- $n$ interior (6), where radiative recombination could occur. 

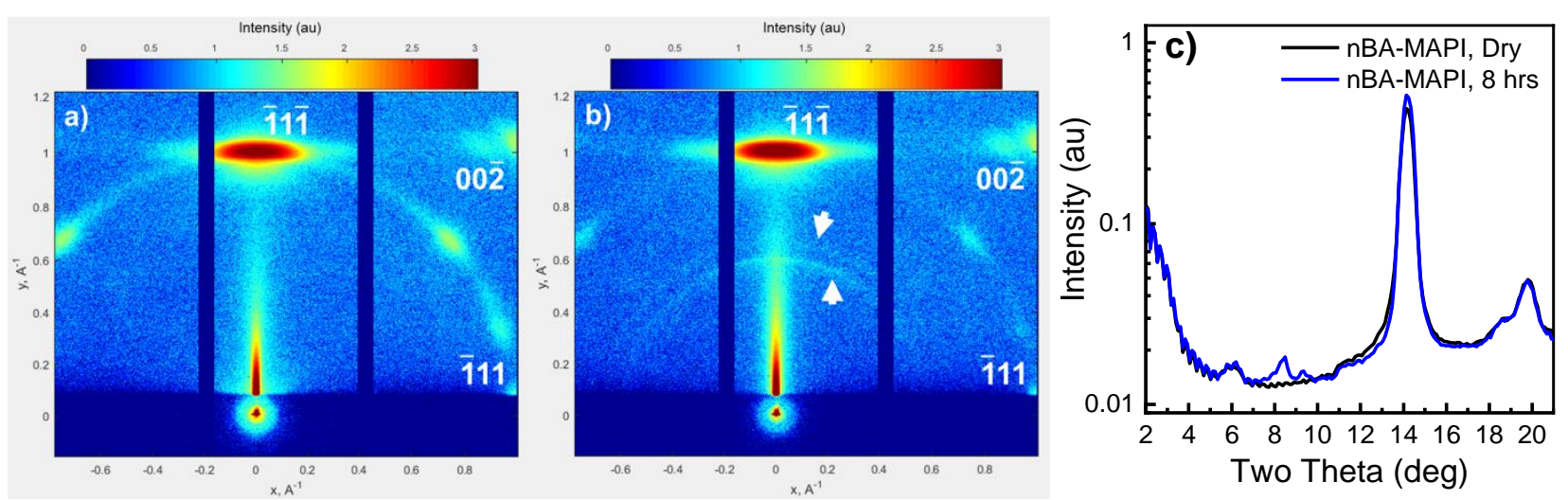

Figure 11: Wide angle $x$-ray scattering spectra for a) fresh and b) 8 hour-exposed nBA-MAPI films on FTO. Bragg diffraction spots for a well-oriented perovskite lattice have been assigned based on previous reports. Following exposure, two diffraction rings (marked with white arrows) appear, indicative of the formation of randomlyoriented low- $n$ perovskites in the film. c) intensity plot vs two theta generated from the WAXS spectra, clearly showing the formation of the two peaks near $8-9^{\circ}$ two theta which can be indexed to $n=2,4$ phases. The small feature near $6^{\circ}$ is an instrumental artifact, and is independent of the film. 


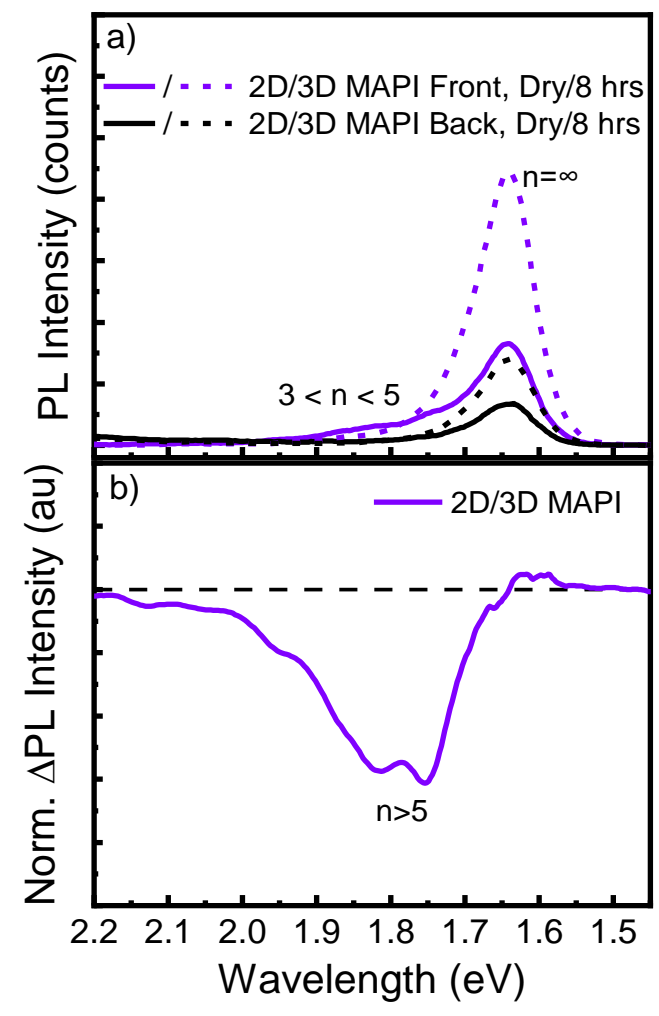

Figure S12: a) Fluorescence spectra for the front and back of a bare 2D/3D MAPI film, before and after exposure to $78 \%$ relative humidity for 8 hours. The primary peak is at $1.65 \mathrm{eV}$, and a broad shoulder near $1.8 \mathrm{eV}$ indicates the presence of nBA-MAPI phases. b) Normalized difference plot for the front of a 2D/3D MAPI film, showing the loss of the shoulder near $1.8 \mathrm{eV}$ following exposure. 

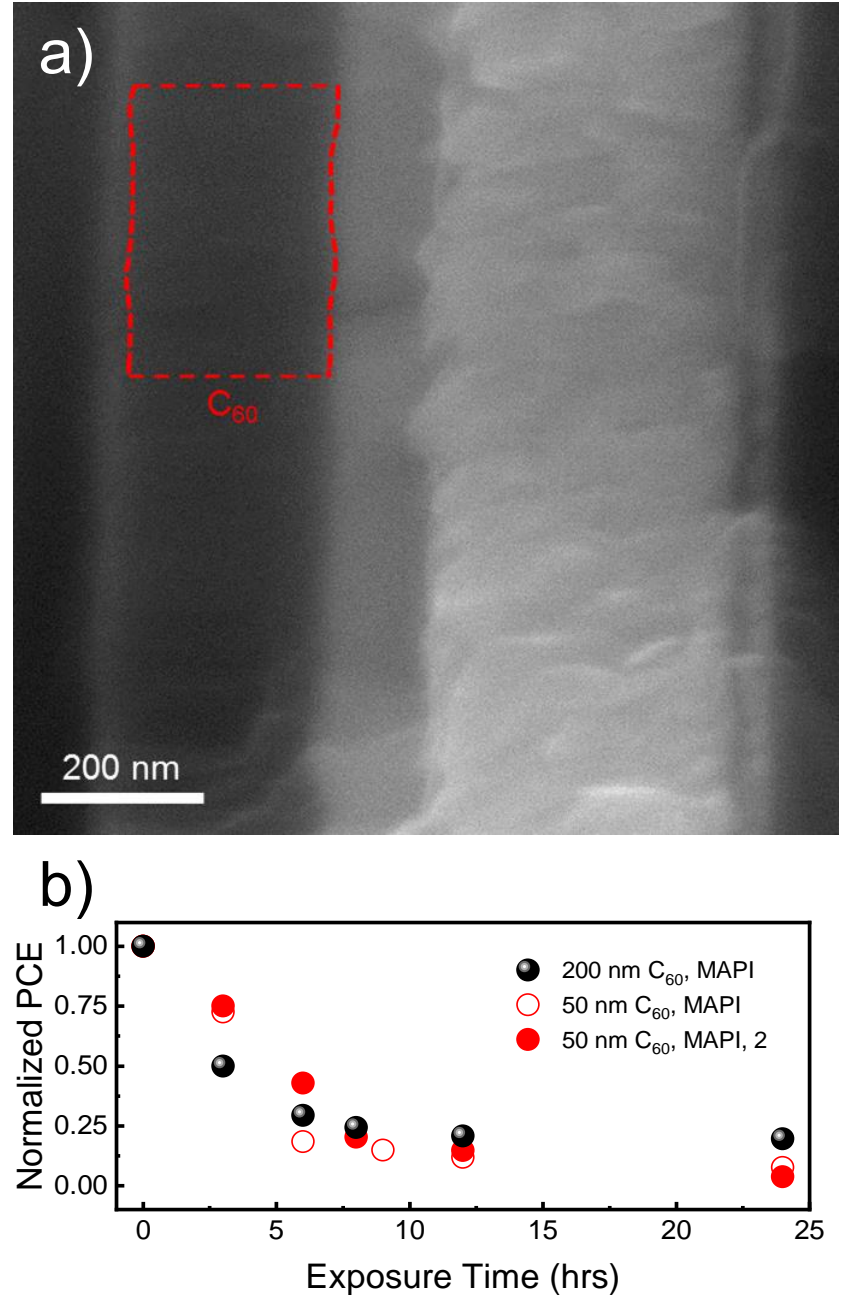

Figure S13: a) cross sectional SEM image of a $200 \mathrm{~nm} \mathrm{C}_{60}$ MAPI device, with the $\mathrm{C}_{60}$ layer outlined in red. b) Normalized PCE for two batches of our typical $50 \mathrm{~nm} \mathrm{C}_{60}$ MAPI devices (red) and the $200 \mathrm{~nm} \mathrm{C}_{60}$ device (black). 


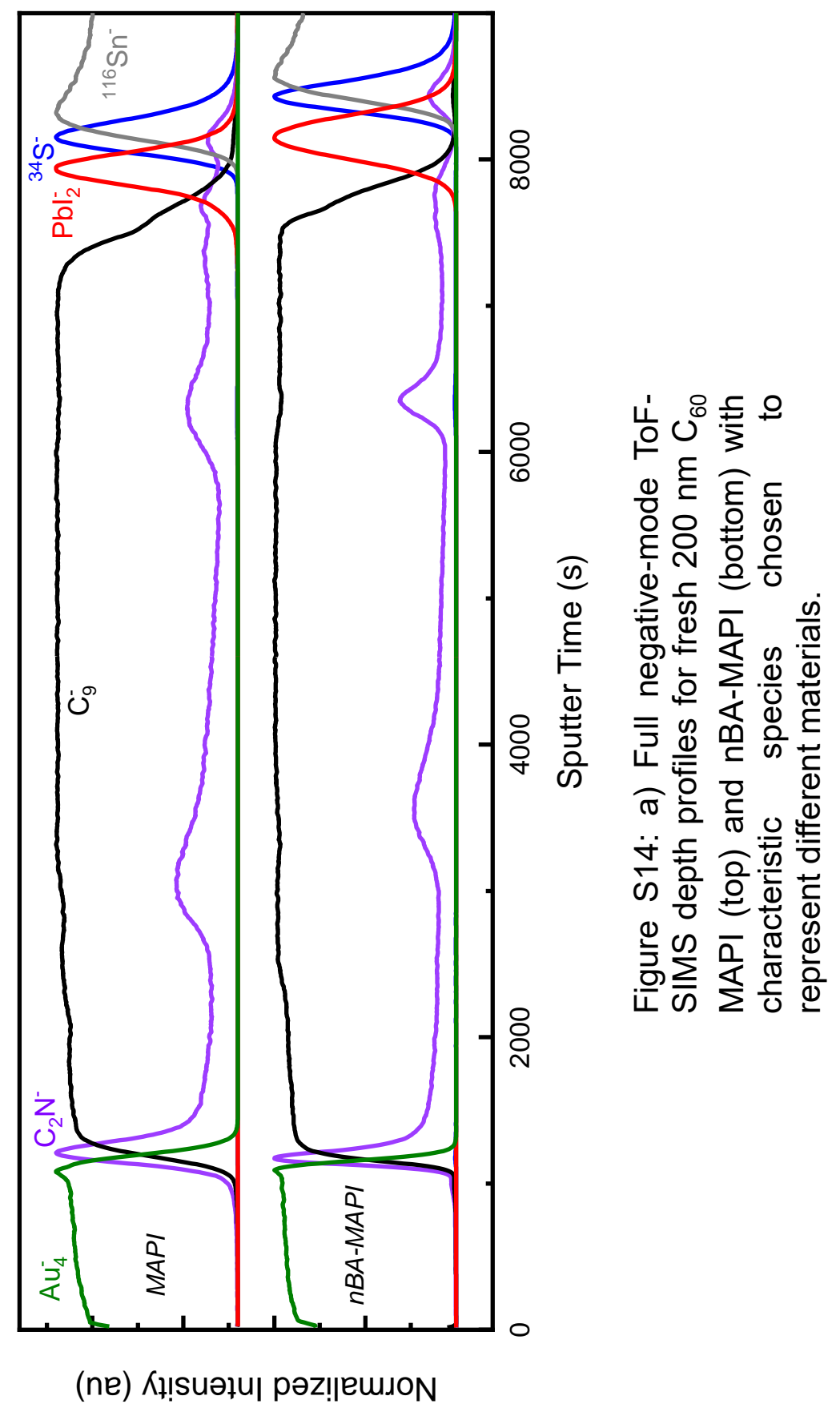



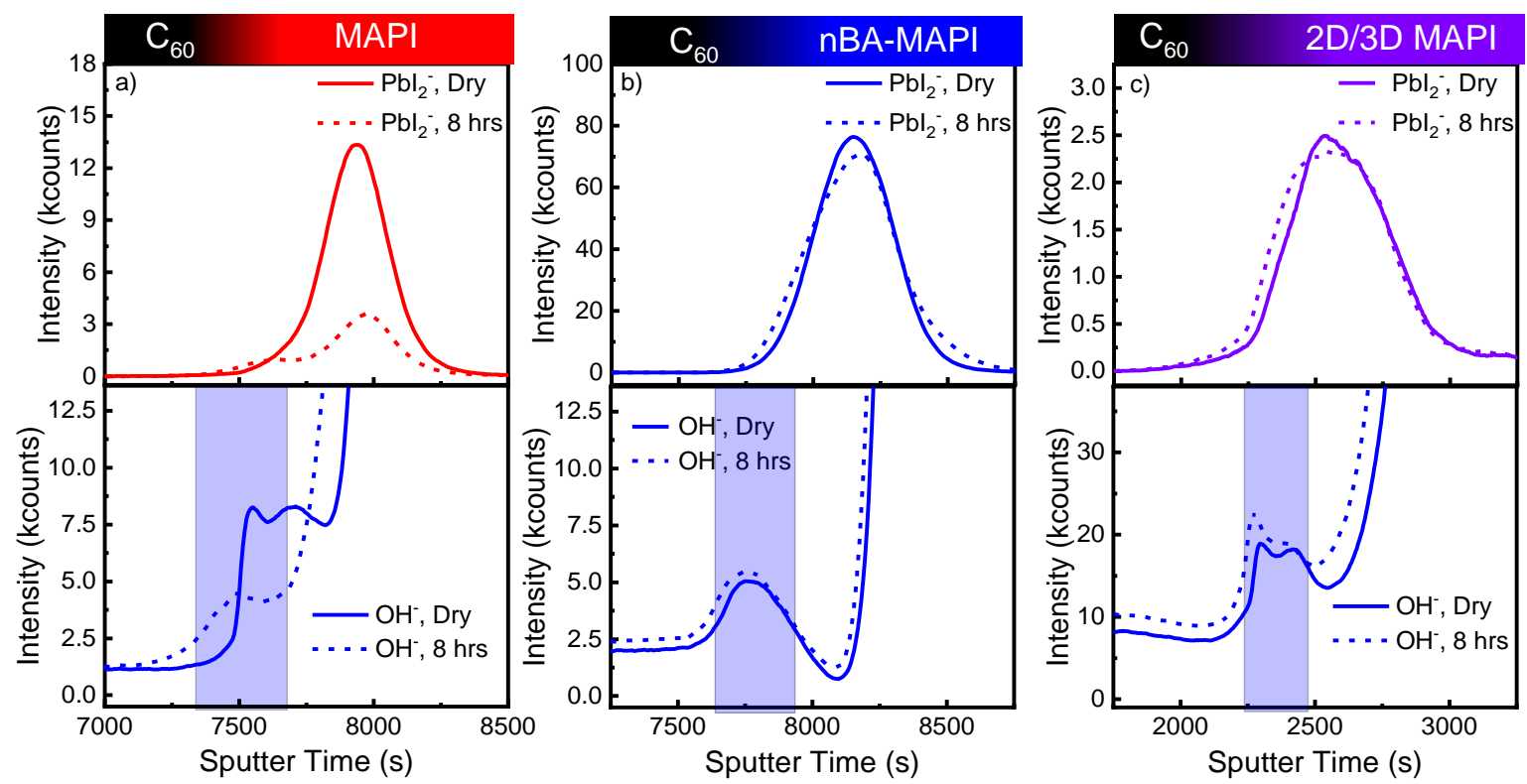

Figure S15: Negative-mode ToF-SIMS depth profiles of (a) MAPI, (b), nBA-MAPI, and (c) 2D/3D MAPI devices, before and after exposure to humidity, near the $\mathrm{C}_{60}$ /perovskite interface; the blue box represents the hydration region in each device. The top profiles show the changes to the perovskite $\left(\mathrm{Pbl}_{2}{ }^{-}\right)$with exposure; nBA-MAPI and 2D/3D MAPI show little change, while MAPI shows the formation of a new peak in the hydration region and a significant decrease in intensity due to hydration damage. The bottom profiles show changes to the hydrated species $\left(\mathrm{OH}^{-}\right)$before and after exposure to humidity. The FWHM of the $\mathrm{OH}$ - peak after exposure is used to determine the hydration region (blue). nBA-MAPI (b) and 2D/3D MAPI (c) show only a slight increase in $\mathrm{OH}^{-}$, while MAPI shows a large decrease, indicating loss of hydrated species with exposure. 


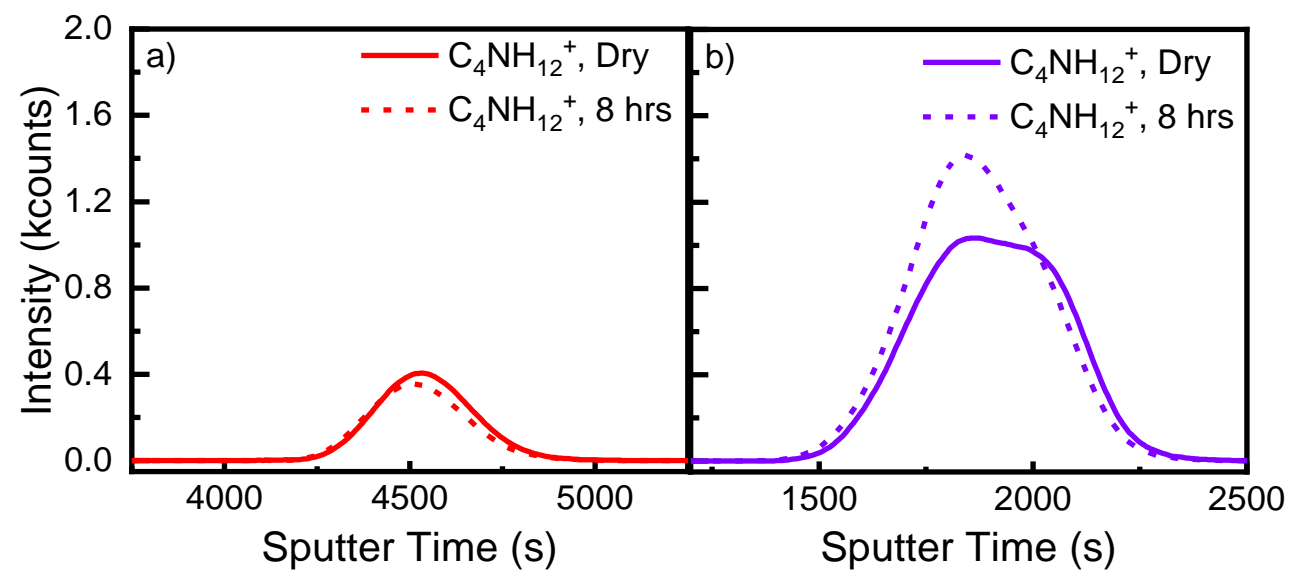

Figure S16: Positive-mode ToF-SIMS depth profiles for the $\mathrm{C}_{4} \mathrm{NH}_{12}{ }^{+}$species representing $n$-butylammonium in (a) MAPI and (b) 2D/3D MAPI devices. The larger signal and dynamic nature of the signal with humidity in b) indicates that the signal is likely a product of $n$-butylammonium; the peak near $1750 \mathrm{~s}$ after exposure agrees with the formation of an nBA-MAPI layer following hydration. 

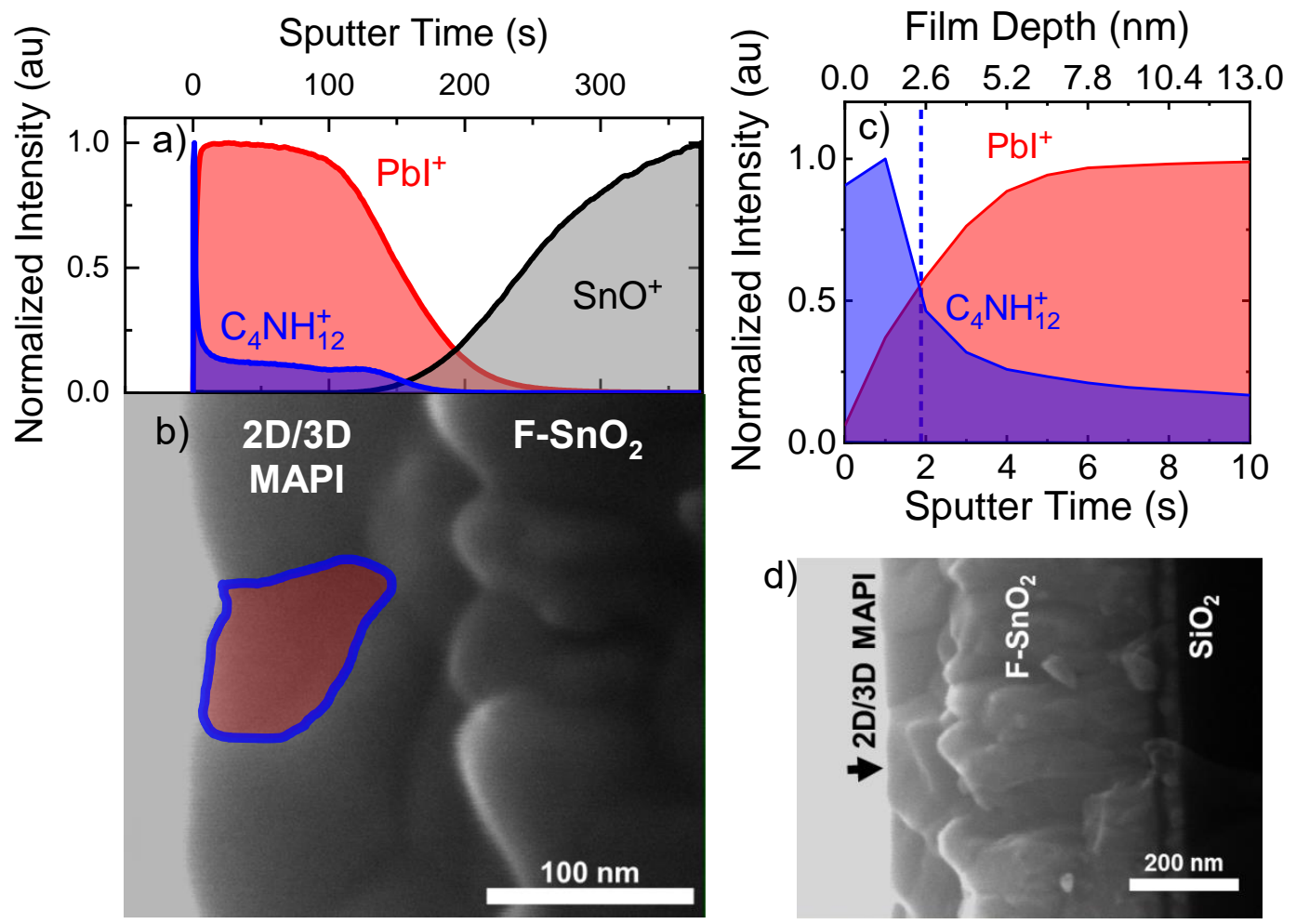

Figure S17: a) Positive mode ToF-SIMS depth profiles for $\mathrm{C}_{4} \mathrm{NH}_{12}{ }^{+}$ (representing $n$-butylammonium), $\mathrm{Pbl}^{+}$(representing 3D MAPI), and $\mathrm{SnO}^{+}$(representing FTO) for a bare 2D/3D MAPI film, showing a sharp $n$-butylammonium peak at the surface of the film that tapers off into the film. b) Corresponding cross sectional SEM image of a 2D/3D MAPI film on FTO. The blue lines represent the nBA-MAPI at the film surface and the coating on the grain boundaries of the 3D MAPI crystals (red). c) Depth profile of the first 10 seconds $(\sim 13 \mathrm{~nm})$ of the 2D/3D MAPI film, showing a nBAMAPI film thickness of roughly $2.5 \mathrm{~nm}$. d) Full cross sectional SEM image of the 2D/3D MAPI film. 


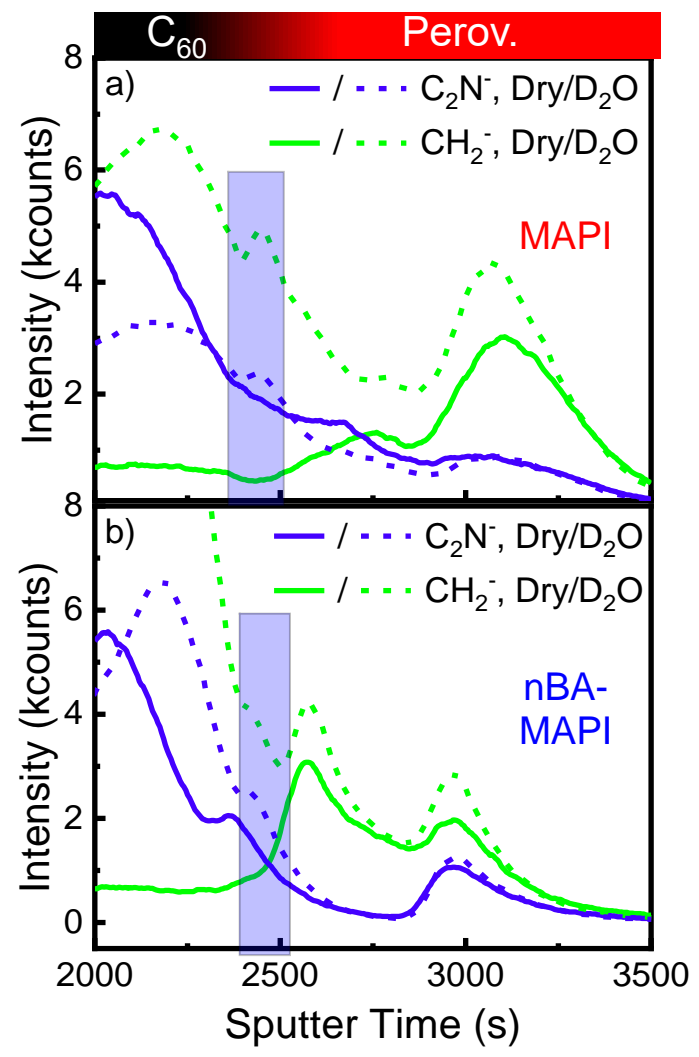

Figure S18: ToF-SIMS depth profiles of $\mathrm{N}$ - and $\mathrm{H}$-containing $\left(\mathrm{C}_{2} \mathrm{~N}^{-}\right.$and $\mathrm{CH}_{2}^{-}$, respectively) organic species in (a) MAPI and (b) nBA-MAPI devices following exposure to $\mathrm{D}_{2} \mathrm{O}$ vapor near the $\mathrm{C}_{60}$ /Perovskite interface; the blue box represents the hydration region. The MAPI sample shows clear formation of a peak in both species inside the hydration region, in agreement with the formation of degraded hydrated-MAPI species at the interface. 

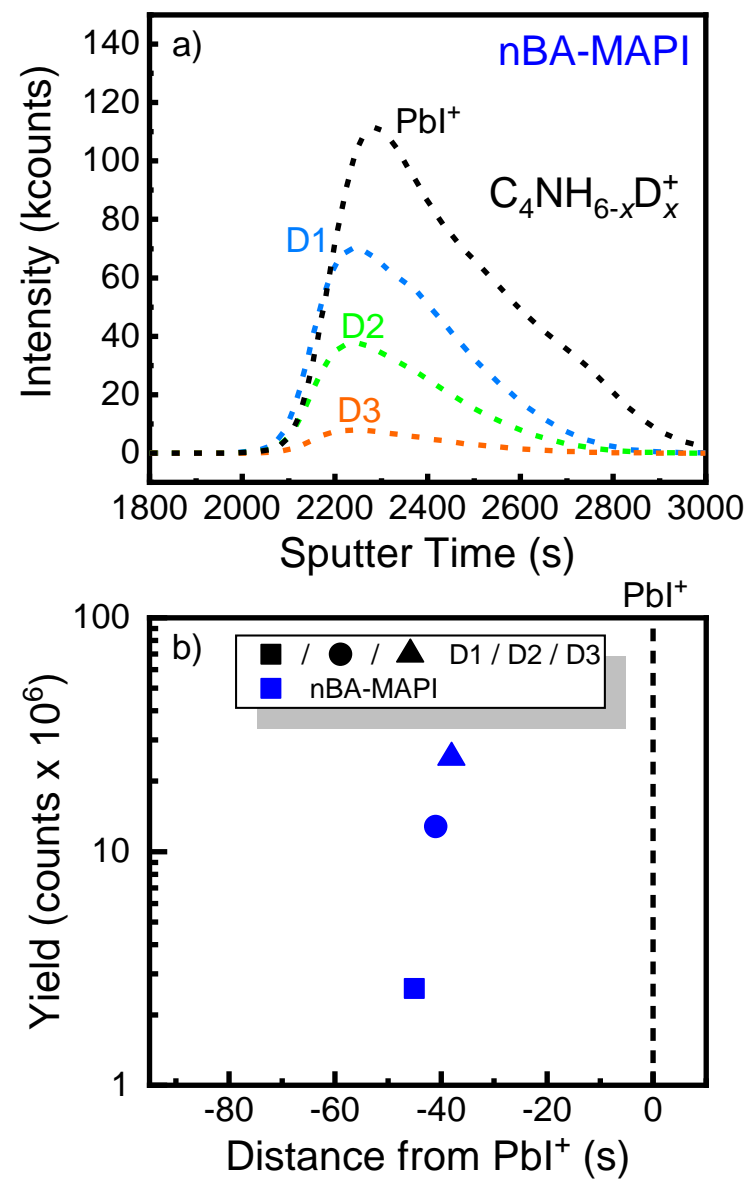

Figure S19: a) Deuterated $n$ butylammonium derivatives $(D=1-3$, i.e. $\mathrm{D} 1=\mathrm{C}_{4} \mathrm{NH}_{11} \mathrm{D}^{+}$) and $\mathrm{Pbl}^{+}$for an nBAMAPI device exposed to $D_{2} O$ vapor for 1 hour. The total ion counts, and therefore abundance, of the derivatives decreases as the level of deuteration increases. b) Total integrated yield of the deuterated $n$ butylammonium derivatives as a function of distance of each derivative's peak from the $\mathrm{Pbl}^{+}$peak $(\mathrm{t}=0)$. The relatively large distance from the center of the perovskite layer indicates a low degree of water penetration into the material. 


\section{$\underline{\text { Supplemental References }}$}

(1) Quan, L. N.; Yuan, M.; Comin, R.; Voznyy, O.; Beauregard, E. M.; Hoogland, S.; Buin, A.; Kirmani, A. R.; Zhao, K.; Amassian, A.; Kim, D. H.; Sargent, E. H. Ligand-Stabilized Reduced-Dimensionality Perovskites. J. Am. Chem. Soc. 2016, 138 (8), 2649-2655. https://doi.org/10.1021/jacs.5b11740.

(2) Koh, T. M.; Shanmugam, V.; Guo, X.; Lim, S. S.; Filonik, O.; Herzig, E. M.; MüllerBuschbaum, P.; Swamy, V.; Chien, S. T.; Mhaisalkar, S. G.; Mathews, N. Enhancing Moisture Tolerance in Efficient Hybrid 3D/2D Perovskite Photovoltaics. J. Mater. Chem. A 2018, 2122-2128. https://doi.org/10.1039/C7TA09657G.

(3) Qing, J.; Liu, X. K.; Li, M.; Liu, F.; Yuan, Z.; Tiukalova, E.; Yan, Z.; Duchamp, M.; Chen, S.; Wang, Y.; Bai, S.; Liu, J. M.; Snaith, H. J.; Lee, C. S.; Sum, T. C.; Gao, F. Aligned and Graded Type-II Ruddlesden-Popper Perovskite Films for Efficient Solar Cells. Adv. Energy Mater. 2018, 8 (21), 1-8. https://doi.org/10.1002/aenm.201800185.

(4) Zhou, N.; Shen, Y.; Li, L.; Tan, S.; Liu, N.; Zheng, G.; Chen, Q.; Zhou, H. Exploration of Crystallization Kinetics in Quasi Two-Dimensional Perovskite and High Performance Solar Cells. J. Am. Chem. Soc. 2018, 140 (1), 459-465. https://doi.org/10.1021/jacs.7b11157.

(5) Tsai, H.; Nie, W.; Blancon, J.-C.; Stoumpos, C. C.; Asadpour, R.; Harutyunyan, B.; Neukirch, A. J.; Verduzco, R.; Crochet, J. J.; Tretiak, S.; Pedesseau, L.; Even, J.; Alam, M. A.; Gupta, G.; Lou, J.; Ajayan, P. M.; Bedzyk, M. J.; Kanatzidis, M. G.; Mohite, A. D. High-Efficiency Two-Dimensional Ruddlesden-Popper Perovskite Solar Cells. Nature 2016, 536 (7616), 312-316. https://doi.org/10.1038/nature18306.

(6) Lin, Y.; Bai, Y.; Fang, Y.; Chen, Z.; Yang, S.; Zheng, X.; Tang, S.; Liu, Y.; Zhao, J.; Huang, J. Enhanced Thermal Stability in Perovskite Solar Cells by Assembling 2D/3D Stacking Structures. J. Phys. Chem. Lett. 2018, 9 (3), 654-658. https://doi.org/10.1021/acs.jpclett.7b02679.

(7) Proppe, A. H.; Quintero-Bermudez, R.; Tan, H.; Voznyy, O.; Kelley, S. O.; Sargent, E. H. Synthetic Control over Quantum Well Width Distribution and Carrier Migration in LowDimensional Perovskite Photovoltaics. J. Am. Chem. Soc. 2018, 140 (8), 2890-2896. https://doi.org/10.1021/jacs.7b12551.

(8) Bouduban, M. E. F.; Burgos-Caminal, A.; Ossola, R.; Teuscher, J.; Moser, J. E. Energy and Charge Transfer Cascade in Methylammonium Lead Bromide Perovskite Nanoparticle Aggregates. Chem. Sci. 2017, 8 (6), 4371-4380. https://doi.org/10.1039/c6sc05211h.

(9) Yuan, M.; Quan, L. N.; Comin, R.; Walters, G.; Sabatini, R.; Voznyy, O.; Hoogland, S.; Zhao, Y.; Beauregard, E. M.; Kanjanaboos, P.; Lu, Z.; Kim, D. H.; Sargent, E. H. Perovskite Energy Funnels for Efficient Light-Emitting Diodes. Nat. Nanotechnol. 2016, 11 (10), 872-877. https://doi.org/10.1038/nnano.2016.110. 
(10) Liu, J.; Leng, J.; Wu, K.; Zhang, J.; Jin, S. Observation of Internal Photoinduced Electron and Hole Separation in Hybrid Two-Dimentional Perovskite Films. J. Am. Chem. Soc. 2017, 139 (4), 1432-1435. https://doi.org/10.1021/jacs.6b12581.

(11) Wang, J.; Leng, J.; Liu, J.; He, S.; Wang, Y.; Wu, K.; Jin, S. Engineered Directional Charge Flow in Mixed Two-Dimensional Perovskites Enabled by Facile CationExchange. J. Phys. Chem. C 2017, 121 (39), 21281-21289. https://doi.org/10.1021/acs.jpcc.7b08535.

(12) Li, L.; Zhou, N.; Chen, Q.; Shang, Q.; Zhang, Q.; Wang, X.; Zhou, H. Unraveling the Growth of Hierarchical Quasi-2D/3D Perovskite and Carrier Dynamics. J. Phys. Chem. Lett. 2018, 9 (5), 1124-1132. https://doi.org/10.1021/acs.jpclett.7b03294.

(13) Stoumpos, C. C.; Cao, D. H.; Clark, D. J.; Young, J.; Rondinelli, J. M.; Jang, J. I.; Hupp, J. T.; Kanatzidis, M. G. Ruddlesden-Popper Hybrid Lead Iodide Perovskite 2D Homologous Semiconductors. Chem. Mater. 2016, 28 (8), 2852-2867. https://doi.org/10.1021/acs.chemmater.6b00847.

(14) Cao, D. H.; Stoumpos, C. C.; Farha, O. K.; Hupp, J. T.; Kanatzidis, M. G. 2D Homologous Perovskites as Light-Absorbing Materials for Solar Cell Applications. J. Am. Chem. Soc. 2015, 137 (24), 7843-7850. https://doi.org/10.1021/jacs.5b03796.

(15) Silver, S.; Dai, Q.; Li, H.; Brédas, J. L.; Kahn, A. Quantum Well Energetics of an n = 2 Ruddlesden-Popper Phase Perovskite. Adv. Energy Mater. 2019, 1901005, 1-7. https://doi.org/10.1002/aenm.201901005.

(16) Chen, Z.; Li, Z.; Zhang, C.; Jiang, X. F.; Chen, D.; Xue, Q.; Liu, M.; Su, S.; Yip, H. L.; Cao, Y. Recombination Dynamics Study on Nanostructured Perovskite Light-Emitting Devices. Adv. Mater. 2018, 30 (38), 1-9. https://doi.org/10.1002/adma.201801370.

(17) Shang, Q.; Wang, Y.; Zhong, Y.; Mi, Y.; Qin, L.; Zhao, Y.; Qiu, X.; Liu, X.; Zhang, Q. Unveiling Structurally Engineered Carrier Dynamics in Hybrid Quasi-Two-Dimensional Perovskite Thin Films toward Controllable Emission. J. Phys. Chem. Lett. 2017, 8 (18), 4431-4438. https://doi.org/10.1021/acs.jpclett.7b01857.

(18) Quan, L. N.; Zhao, Y.; García De Arquer, F. P.; Sabatini, R.; Walters, G.; Voznyy, O.; Comin, R.; Li, Y.; Fan, J. Z.; Tan, H.; Pan, J.; Yuan, M.; Bakr, O. M.; Lu, Z.; Kim, D. H.; Sargent, E. H. Tailoring the Energy Landscape in Quasi-2D Halide Perovskites Enables Efficient Green-Light Emission. Nano Lett. 2017, 17 (6), 3701-3709. https://doi.org/10.1021/acs.nanolett.7b00976.

(19) Proppe, A. H.; Elkins, M. H.; Voznyy, O.; Pensack, R. D.; Zapata, F.; Besteiro, L. V.; Quan, L. N.; Quintero-Bermudez, R.; Todorovic, P.; Kelley, S. O.; Govorov, A. O.; Gray, S. K.; Infante, I.; Sargent, E. H.; Scholes, G. D. Spectrally Resolved Ultrafast Exciton Transfer in Mixed Perovskite Quantum Wells. J. Phys. Chem. Lett. 2019, 10 (3), 419-426. https://doi.org/10.1021/acs.jpclett.9b00018.

(20) Liu, J.; Leng, J.; Wu, K.; Zhang, J.; Jin, S. Observation of Internal Photoinduced Electron and Hole Separation in Hybrid Two-Dimentional Perovskite Films. J. Am. Chem. Soc. 
2017, 139 (4), 1432-1435. https://doi.org/10.1021/jacs.6b12581.

(21) Silver, S.; Yin, J.; Li, H.; Brédas, J. L.; Kahn, A. Characterization of the Valence and Conduction Band Levels of $\mathrm{n}=1$ 2D Perovskites: A Combined Experimental and Theoretical Investigation. Adv. Energy Mater. 2018, 8 (16), 1-7. https://doi.org/10.1002/aenm.201703468.

(22) Endres, J.; Egger, D. A.; Kulbak, M.; Kerner, R. A.; Zhao, L.; Silver, S. H.; Hodes, G.; Rand, B. P.; Cahen, D.; Kronik, L.; Kahn, A. Valence and Conduction Band Densities of States of Metal Halide Perovskites: A Combined Experimental-Theoretical Study. J. Phys. Chem. Lett. 2016, 7 (14), 2722-2729. https://doi.org/10.1021/acs.jpclett.6b00946.

(23) Feron, K.; Nagle, T. J.; Rozanski, L. J.; Gong, B. B.; Fell, C. J. Spatially Resolved Photocurrent Measurements of Organic Solar Cells: Tracking Water Ingress at Edges and Pinholes. Sol. Energy Mater. Sol. Cells 2013, 109, 169-177. https://doi.org/10.1016/j.solmat.2012.10.027. 